\title{
A QCD Sum Rule Approach to the Charmonium - Pion Cross Section
}

\author{
M. Nielsen, F.S. Navarra, \\ Instituto de Física, Universidade de São Paulo \\ C.P. 66318, 05315-970 São Paulo, SP, Brazil \\ G. Krein, and R.S. Marques de Carvalho \\ Instituto de Física Teórica, Universidade Estadual Paulista \\ Rua Pamplona 145, 01405-900 São Paulo, SP, Brazil
}

Received on 30 October, 2002

\begin{abstract}
We study the Borel sum rule for the tensor amplitude of the processes $J / \psi \pi \rightarrow \bar{D} D^{*}, \bar{D} D, \bar{D}^{*} D^{*}$ and $\bar{D} D^{*}$. We also evaluate the cross sections as a function of $\sqrt{s}$. We find that our results are smaller than the $J / \psi \pi \rightarrow$ charmed mesons cross sections obtained with models based on meson exchange, but are close to those obtained with quark exchange models.
\end{abstract}

\section{Introduction}

In relativistic heavy ion collisions $J / \psi$ suppression has been recognized as an important tool to identify the possible phase transition to quark-gluon plasma (QGP). Matsui and Satz [1] predicted that in presence of quark-gluon plasma, binding of a $c \bar{c}$ pair into a $J / \psi$ meson will be hindered, leading to the so called $J / \psi$ suppression in heavy ion collisions. Over the years several experiments measured the $J / \psi$ yield in heavy ion collisions (for a review of data and interpretations see Ref.[2, 3]). In brief, experimental data do show suppression. However, this could be attributed to more conventional $J / \psi$ absorption by comovers, not present in $\mathrm{pA}$ collisions. In heavy ion collisions, part of the charmonium interactions happens in the early stages of the collisions and, therefore, at high energies $(\sqrt{s} \simeq 10-20 \mathrm{GeV})$ and one may try to apply perturbative QCD. However, even in this regime, nonperturbative effects may be important [4]. On the other hand, a significant part of the charmonium - hadron interactions occurs when other light particles have already been produced. This kind of interactions happen at much lower energies $(\sqrt{s} \leq 5 \mathrm{GeV})$ and one has to apply nonperturbative methods. In this work we use the QCD sum rules (QCDSR) technique $[5,6]$ to study the $J / \psi-\pi$ dissociation. In view of our relatively poor understanding of $J / \psi$ reactions in nuclear matter and considering the large discrepan- cies between different model estimates, we believe that our work adds to a better understanding of this important topic.

\section{The QCDSR Calculation}

Let us start with the the four-point function for the process $J / \psi \pi \rightarrow \bar{D} D^{*}:$

$$
\begin{aligned}
\Pi_{\mu \nu} & =i \int d^{4} x d^{4} y d^{4} z e^{-i p_{1} \cdot x} e^{-i p_{2} .0} e^{i p_{3} \cdot y} e^{i p_{4} . z} \\
& \times\left\langle 0\left|T\left\{j_{\pi}(x) j_{\nu}^{D^{*}}(y) j_{\mu}^{\psi}(0) j_{D}(z)\right\}\right| 0\right\rangle
\end{aligned}
$$

with the currents given by $j_{\pi}=\bar{d} i \gamma_{5} u, j_{\nu}^{D^{*}}=\bar{u} \gamma_{\nu} c$, $j_{\mu}^{\psi}=\bar{c} \gamma_{\mu} c$ and $j_{D}=\bar{c} i \gamma_{5} d[6]$, where $c, u$ and $d$ are the charm, up and down quark fields respectively, and $p_{1}, p_{2}, p_{3}$ and $p_{4}$ are the four-momenta of the mesons $\pi, J / \psi, D^{*}$ and $D$ respectively, with $p_{1}+p_{2}=p_{3}+p_{4}$.

Following Reinders, Rubinstein, and Yazaki [6], and others $[7,8,9]$, we can write a sum rule valid only at $p_{1}^{2}=0$ (at the pion pole if one neglects the pion mass). The perturbative diagram does not contribute with $1 / p_{1}^{2}$ and, up to dimension four, only the diagrams proportional to the quark condensate contribute. After collecting the $1 / p_{1}^{2}$ terms on the theoretical side and taking the limit $p_{1 \mu} \rightarrow 0$ in the residue of the pion pole, one obtains:

$$
\Pi_{\mu \nu}^{<\bar{q} q>}=-\frac{2 m_{c}\langle\bar{q} q\rangle}{p_{1}^{2}} \frac{p_{1 \nu}\left(p_{1 \mu}+p_{2 \mu}-2 p_{3 \mu}\right)-p_{1 \mu} p_{2 \nu}}{\left(p_{3}^{2}-m_{c}^{2}\right)\left(p_{4}^{2}-m_{c}^{2}\right)}
$$


The phenomenological side of the correlation function, $\Pi_{\mu \nu}$, is obtained by the consideration of $J / \psi, \pi, D$ and $D^{*}$ state contribution to the matrix element in Eq. (1):

$$
\begin{gathered}
\Pi_{\mu \nu}^{p h e n}=-\frac{m_{\pi}^{2} f_{\pi}}{m_{u}+m_{d}} \frac{m_{D}^{2} f_{D}}{m_{c}} \frac{m_{D^{*}} f_{D^{*}} m_{\psi} f_{\psi} \mathcal{M}^{\alpha \beta}}{\left(p_{1}^{2}-m_{\pi}^{2}\right)\left(p_{4}^{2}-m_{D}^{2}\right)} \\
\times \frac{g_{\mu \alpha}-p_{2 \mu} p_{2 \alpha} / m_{\psi}^{2}}{p_{2}^{2}-m_{\psi}^{2}} \frac{g_{\nu \beta}-p_{3 \nu} p_{3 \beta} / m_{D^{*}}^{2}}{p_{3}^{2}-m_{D^{*}}^{2}}+\text { h. r. },
\end{gathered}
$$

where h. r. means higher resonances and the hadronic amplitude for the process $J / \psi \pi \rightarrow \bar{D} D^{*}$ is given by

$$
\mathcal{M}=\mathcal{M}_{\mu \nu}\left(p_{1}, p_{2}, p_{3}, p_{4}\right) \epsilon_{2}^{\mu} \epsilon_{3}^{* \nu} .
$$

We note that one has $1 / p_{1}^{2}$ pole in Eq. (3) in the limit of a vanishing pion mass. Contracting the hadronic amplitude with the numerators of $J / \psi$ and $D^{*}$ propagators in Eq. (3) and comparing with Eq. (2), the structure defining $\mathcal{M}_{\mu \nu}$ in Eq. (4) is easily identified. Therefore, defining

$$
\mathcal{M}_{\mu \nu}=\Lambda_{D D^{*}}\left(p_{1 \mu} p_{1 \nu}-p_{1 \mu} p_{2 \nu}-2 p_{1 \nu} p_{3 \mu}\right)
$$

we can write a sum rule for $\Lambda_{D D^{*}}$ in any of the three structures appearing in Eq. (5). To improve the matching between the phenomenological and theoretical sides we follow the usual procedure and make a single Borel transformation to all the external momenta (except $p_{1}^{2}$ ) taken to be equal: $-p_{2}^{2}=-p_{3}^{2}=-p_{4}^{2}=P^{2} \rightarrow M^{2}$. The problem of doing a single Borel transformation is the fact that terms associated with the pole-continuum transitions are not suppressed [10]. In ref. [10] it was explicitly shown that the pole-continuum transition has a different behavior as a function of the Borel mass as compared with the double pole contribution (triple pole contribution in our case) and continuum contribution: it grows with $M^{2}$ as compared with the contribution of the fundamental states. Therefore, the polecontinuum contribution can be taken into account through the introduction of a parameter $A_{D D^{*}}$ in the phenomenological side of the sum rule $[8,9,10]$. Thus, neglecting $m_{\pi}^{2}$ in the denominator of Eq. (3) and doing a single Borel transform in $-p_{2}^{2}=-p_{3}^{2}=-p_{4}^{2}=P^{2}$, we get

$$
\begin{aligned}
& \frac{\Lambda_{D D^{*}}+A_{D D^{*}} M^{2}}{m_{D^{*}}^{2}-m_{\psi}^{2}}\left[\frac{e^{-m_{D}^{2} / M^{2}}-e^{-m_{\psi}^{2} / M^{2}}}{m_{\psi}^{2}-m_{D}^{2}}-\left(\psi \rightarrow D^{*}\right)\right] \\
& =-2 m_{c}\langle\bar{q} q\rangle \frac{e^{-m_{c}^{2} / M^{2}}}{M^{2}} \frac{m_{c}\left(m_{u}+m_{d}\right)}{m_{\pi}^{2} m_{D}^{2} m_{D^{*}} m_{\psi} f_{\pi} f_{D} f_{D^{*}} f_{\psi}}
\end{aligned}
$$

where we have transferred to the theoretical side the couplings of the currents with the mesons, and have introduced, in the phenomenological side, the parameter $A_{D D^{*}}$ to account for possible nondiagonal transitions.

For consistency we use in our analysis the QCDSR expressions for the decay constants of the $J / \psi, D^{*}$ and $D$ mesons up to dimension four in lowest order of $\alpha_{s}$ :

$$
\begin{gathered}
f_{D}^{2}=\frac{3 m_{c}^{2}}{8 \pi^{2} m_{D}^{4}} \int_{m_{c}^{2}}^{u_{D}} d u \frac{\left(u-m_{c}^{2}\right)^{2}}{u} e^{\left(m_{D}^{2}-u\right) / M_{M}^{2}}-\frac{m_{c}^{3}}{m_{D}^{4}}\langle\bar{q} q\rangle e^{\left(m_{D}^{2}-m_{c}^{2}\right) / M_{M}^{2}} \\
f_{D^{*}}^{2}=\frac{1}{8 \pi^{2} m_{D^{*}}^{2}} \int_{m_{c}^{2}}^{u_{D *}} d u \frac{\left(u-m_{c}^{2}\right)^{2}}{u}\left(2+\frac{m_{c}^{2}}{u}\right) e^{\left(m_{D^{*}}^{2}-u\right) / M_{M}^{2}}-\frac{m_{c}}{m_{D^{*}}^{2}}\langle\bar{q} q\rangle e^{\left(m_{D^{*}}^{2}-m_{Q}^{2}\right) / M_{M}^{2}} \\
f_{\psi}^{2}=\frac{1}{4 \pi^{2}} \int_{4 m_{c}^{2}}^{u_{\psi}} d u \frac{\left(u+2 m_{c}^{2}\right) \sqrt{u-4 m_{c}^{2}}}{u^{3 / 2}} e^{\left(m_{\psi}^{2}-u\right) / M_{M}^{2}}
\end{gathered}
$$

where $M_{M}^{2}$ represents the Borel mass in the two-point function, $u_{M}$ is for the continuum threshold for the meson $M$, and we have omitted the numerically insignificant contribution of the gluon condensate.

The parameter values used in all calculations are $m_{u}+$ $m_{d}=14 \mathrm{MeV}, m_{c}=1.5 \mathrm{GeV}, m_{\pi}=140 \mathrm{MeV}, m_{D}=$ $1.87 \mathrm{GeV}, m_{D^{*}}=2.01 \mathrm{GeV}, m_{\psi}=3.097 \mathrm{GeV}, f_{\pi}=$ 131.5 MeV, $\langle\bar{q} q\rangle=-(0.23)^{3} \mathrm{GeV}^{3}$. We parametrize the continuum thresholds as $u_{M}=\left(m_{M}+\Delta_{u}\right)^{2}$. Using the Borel region $3 \leq M_{M}^{2} \leq 6 \mathrm{GeV}^{2}$ for the $D^{*}$ and $D$ mesons 
and $6 \leq M_{M}^{2} \leq 10 \mathrm{GeV}^{2}$ for the $J / \psi$, we found good stability for $f_{D}, f_{D *}$ and $f_{\psi}$ with $\Delta_{u} \sim 0.6 \mathrm{GeV}$. We obtained $f_{D}=155 \pm 5 \mathrm{MeV}, f_{D^{*}}=195 \pm 5 \mathrm{MeV}$ and $f_{\psi}=225 \pm 10 \mathrm{MeV}$, which are acceptable values for these decay constants .

\section{Results and Discussion}

Using $\Delta_{u}=0.6 \mathrm{GeV}$, we obtain the QCD sum rule results for $\Lambda_{D D^{*}}+A_{D D^{*}} M^{2}$ as a function of $M^{2}$. We find that they follow a straight line in the Borel region $8 \leq M^{2} \leq$ $16 \mathrm{GeV}^{2}$ [11]. The value of the amplitude $\Lambda$ is obtained by the extrapolation of the line to $M^{2}=0[8,9,10]$. Fitting the QCD sum rule results to a straight line we get

$$
\Lambda_{D D^{*}} \simeq 17.71 \mathrm{GeV}^{-2} \text {. }
$$

As expected, in our approach $\Lambda$ is just a number and all dependence of $\mathcal{M}_{\mu \nu}$ (Eq. (5)) on particle momenta is contained in the Dirac structure. This is a consequence of our low energy approximation.

Following the same procedure, we have also considered the processes $J / \psi \pi \rightarrow \bar{D} D$ and $J / \psi \pi \rightarrow \bar{D}^{*} D^{*}$. Similarly to the case $J / \psi \pi \rightarrow \bar{D} D^{*}$, in the OPE side the only diagrams, up to dimension four, contributing with $1 / p_{1}^{2}$ are the quark condensate diagrams. Comparing the phenomenological and OPE sides of the correlators we can identify the structure defining the hadronic amplitudes:

$$
\mathcal{M}_{\mu}=\Lambda_{D D} \epsilon_{\mu \alpha \beta \sigma} p_{1}^{\alpha} p_{3}^{\beta} p_{4}^{\sigma}
$$

and

$$
\begin{aligned}
\mathcal{M}_{\mu \nu \alpha} & =\Lambda_{D^{*} D^{*}}\left[p_{1}^{\beta} p_{3}^{\lambda} p_{4}^{\gamma}\left(-\epsilon_{\nu \beta \lambda \gamma} g_{\alpha \mu}+\epsilon_{\mu \beta \lambda \gamma} g_{\alpha \nu}-\epsilon_{\alpha \beta \lambda \gamma} g_{\mu \nu}\right)+\epsilon_{\mu \nu \beta \lambda}\left(p_{1}^{\beta} p_{4}^{\lambda} p_{3 \alpha}\right.\right. \\
& \left.-p_{3}^{\beta} p_{4}^{\lambda} p_{1 \alpha}\right)+\epsilon_{\alpha \nu \beta \lambda}\left(p_{1}^{\beta} p_{3}^{\lambda} p_{1 \mu}+p_{3}^{\beta} p_{4}^{\lambda} p_{1 \mu}-p_{1}^{\beta} p_{4}^{\lambda} p_{3 \mu}+p_{1}^{\beta} p_{3}^{\lambda} p_{4 \mu}\right) \\
& \left.+\epsilon_{\alpha \mu \beta \lambda}\left(-p_{1}^{\beta} p_{3}^{\lambda} p_{1 \nu}-p_{3}^{\beta} p_{4}^{\lambda} p_{1 \nu}+p_{1}^{\beta} p_{4}^{\lambda} p_{1 \nu}+p_{1}^{\beta} p_{3}^{\lambda} p_{4 \mu}\right)\right] .
\end{aligned}
$$

Performing a single Borel transform in $-p_{2}^{2}=-p_{3}^{2}=-p_{4}^{2}=P^{2}$, we get

$$
\begin{aligned}
& \frac{\Lambda_{M M}+A_{M M} M^{2}}{m_{M}^{2}-m_{\psi}^{2}} f_{M}\left(M^{2}\right)=C_{M} \frac{m_{u}+m_{d}}{m_{\pi}^{2} m_{M}^{2} m_{\psi} f_{\pi} f_{M}^{2} f_{\psi}} \\
& \times 2\langle\bar{q} q\rangle \frac{e^{-m_{c}^{2} / M^{2}}}{M^{2}},
\end{aligned}
$$

where the subscript $M$ stands for the $D$ or $D^{*}$ mesons, with $C_{D}=\frac{m_{c}^{2}}{m_{D}^{2}}, C_{D^{*}}=1$ and

$$
f_{M}\left(M^{2}\right)=\frac{e^{-m_{M}^{2} / M^{2}}}{M^{2}}-\frac{e^{-m_{M}^{2} / M^{2}}-e^{-m_{\psi}^{2} / M^{2}}}{m_{\psi}^{2}-m_{M}^{2}} .
$$

The QCD sum rule results for $\Lambda_{D D}+A_{D D} M^{2}$ and $\Lambda_{D^{*} D^{*}}+A_{D^{*} D^{*}} M^{2}$ as a function of $M^{2}$ are obtained in a similar way and the amplitudes $\Lambda_{D D}$ and $\Lambda_{D^{*} D^{*}}$ are extracted by the extrapolation of the line to $M^{2}=0$. We get [11]:

$$
\Lambda_{D D} \simeq 12.25 \mathrm{GeV}^{-1}, \quad \Lambda_{D^{*} D^{*}} \simeq 11.39 \mathrm{GeV}^{-3} .
$$

Having the QCD sum rule results for the amplitude of the three processes $J / \psi \pi \rightarrow \bar{D} D^{*}, \bar{D} D, \bar{D}^{*} D^{*}$, given in Eqs. (5), (11) and (12) we can evaluate the differential cross section. After including isospin factors, the differential cross section for the $J / \psi \pi$ dissociation is given by

$$
\frac{d \sigma}{d t}=\frac{1}{96 \pi s \mathbf{p}_{i, c m}^{2}} \sum_{\text {spin }}|\mathcal{M}|^{2}
$$

where $\mathbf{p}_{i, c m}$ is the three-momentum of $p_{1}$ (or $p_{2}$ ) in the center of mass frame (with $p_{1}\left(p_{2}\right)$ being the four-momentum of the $\pi(J / \psi))$ :

$$
\mathbf{p}_{i, c m}^{2}=\frac{\lambda\left(s, m_{\pi}^{2}, m_{\psi}^{2}\right)}{4 s},
$$

with $\lambda(x, y, z)=x^{2}+y^{2}+z^{2}-2 x y-2 x z-2 y z, s=\left(p_{1}+p_{2}\right)^{2}, t=\left(p_{1}-p_{3}\right)^{2}$.

In Eq. (16), the sum over the spins of the amplitude squared is given by 


$$
\sum_{\text {spin }}|\mathcal{M}|^{2}=\mathcal{M}_{\mu \nu} \mathcal{M}_{\mu^{\prime} \nu^{\prime}}^{*}\left(g^{\mu \mu^{\prime}}-\frac{p_{2}^{\mu} p_{2}^{\mu^{\prime}}}{m_{\psi}^{2}}\right)\left(g^{\nu \nu^{\prime}}-\frac{p_{3}^{\nu} p_{3}^{\nu^{\prime}}}{m_{D^{*}}^{2}}\right)
$$

for $J / \psi \pi \rightarrow \bar{D} D^{*}$, with $p_{3}\left(p_{4}\right)$ being the four-momentum of $D^{*}(D)$.

$$
\sum_{\text {spin }}|\mathcal{M}|^{2}=\mathcal{M}_{\mu} \mathcal{M}_{\mu^{\prime}}^{*}\left(g^{\mu \mu^{\prime}}-\frac{p_{2}^{\mu} p_{2}^{\mu^{\prime}}}{m_{\psi}^{2}}\right)
$$

for $J / \psi \pi \rightarrow \bar{D} D$, and

$$
\sum_{\text {spin }}|\mathcal{M}|^{2}=\mathcal{M}_{\mu \nu \alpha} \mathcal{M}_{\mu^{\prime} \nu^{\prime} \alpha^{\prime}}^{*}\left(g^{\mu \mu^{\prime}}-\frac{p_{2}^{\mu} p_{2}^{\mu^{\prime}}}{m_{\psi}^{2}}\right)\left(g^{\nu \nu^{\prime}}-\frac{p_{3}^{\nu} p_{3}^{\nu^{\prime}}}{m_{D^{*}}^{2}}\right)\left(g^{\alpha \alpha^{\prime}}-\frac{p_{4}^{\alpha} p_{4}^{\alpha^{\prime}}}{m_{D^{*}}^{2}}\right)
$$

for $J / \psi \pi \rightarrow \bar{D}^{*} D^{*}$.

Using our QCD sum rule results we show, in Fig. 1, the cross section for the $J / \psi \pi$ dissociation. It is important to keep in mind that, since our sum rule was derived in the limit $p_{1} \rightarrow 0$, we can not extend our results to large values of $\sqrt{s}$.

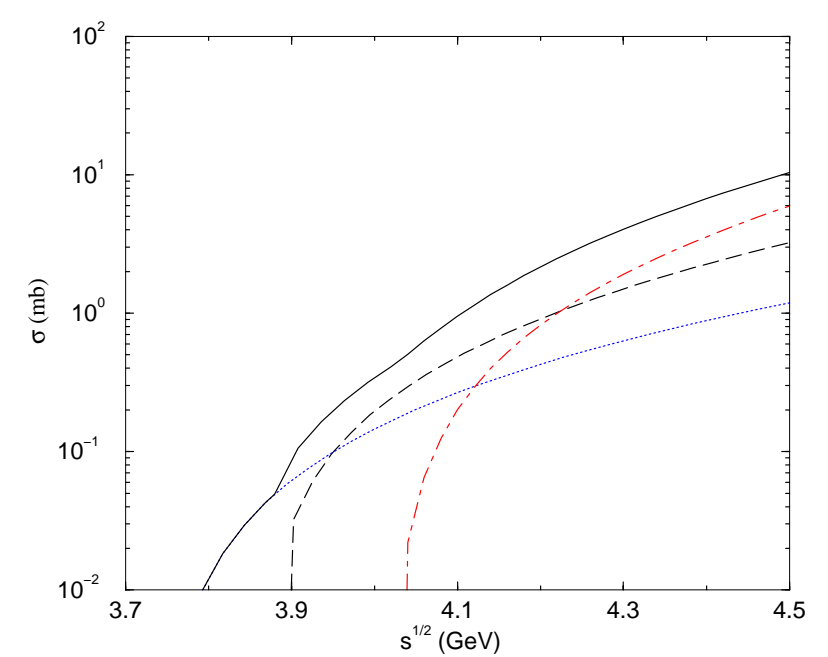

Figure 1. Total cross sections of the processes $J / \psi \pi \rightarrow \bar{D} D^{*}+$ $D \bar{D}^{*}$ (dashed line), $\bar{D} D$ (dotted line) and $\bar{D}^{*} D^{*}$ (dot-dashed line). The solid line gives the total $J / \psi \pi$ dissociation cross section.

Our first conclusion is that our results show that, for values of $\sqrt{s}$ far from the $J / \psi \pi \rightarrow \bar{D}^{*} D^{*}$ threshold, $\sigma_{J / \psi \pi \rightarrow \bar{D}^{*} D^{*}} \geq \sigma_{J / \psi \pi \rightarrow \bar{D} D^{*}+D \bar{D}^{*}} \geq \sigma_{J / \psi \pi \rightarrow \bar{D} D}$, in agreement with calculations using meson exchange models based on effective lagrangians, as discussed, for example, in Ref. [12], but in disagreement with the results obtained with the nonrelativistic quark model of Ref. [13], which show that the state $\bar{D}^{*} D$ has a larger production cross section than $\bar{D}^{*} D^{*}$. Furthermore, our curves indicate that the cross section grows monotonically with the c.m.s. energy but not as fast, near the thresholds, as it does in the calculations in Refs. [12]. Again, this behavior is in opposition to [13], where a peak just after the threshold followed by continuous decrease in the cross section was found.

At higher energies, due to our low energy approxima- tion, our approach gradually looses validity. In the fiducial region, close to threshold, $4.1 \leq \sqrt{s} \leq 4.3 \mathrm{GeV}$, we find $2.5 \leq \sigma \leq 4.0 \mathrm{mb}$ and these values are much smaller than those obtained with the effective Lagrangians without form factors in the hadronic vertices, but agree in order of magnitude with the quark model calculations of [13].

\section{Acknowledgments}

This work was supported by CNPq and FAPESP (contract numbers 98/06590-2, 99/12987-5 and 00/04422- 7).

\section{References}

[1] T. Matsui and H. Satz, Phys. Lett. B178, 416 (1986).

[2] R. Vogt, Phys. Reports, 310, 197 (1999).

[3] C. Gerschel, and J. Huefner, Ann. Rev. Nucl. Pat. Sci. 49, 255 (1999).

[4] H.G. Dosch, F.S. Navarra, M. Nielsen and M. Rueter, Phys. Lett. B466, 363 (1999).

[5] M.A. Shifman, A.I. Vainshtein and V.I. Zakharov, Nucl. Phys. B120, 316 (1977).

[6] L.J. Reinders, H. Rubinstein and S. Yazaki, Phys. Rep. 127, 1 (1985).

[7] S. Choe, M.K. Cheoun and S.H. Lee, Phys. Rev. C53, 1363 (1996).

[8] M.E. Bracco, F.S. Navarra and M. Nielsen, Phys. Lett. B454, 346 (1999).

[9] F.S. Navarra, M. Nielsen, M.E. Bracco, M. Chiapparini and C.L. Schat, Phys. Lett. B489, 319 (2000).

[10] B.L. Ioffe and A.V. Smilga, Nucl. Phys. B232, 109 (1984).

[11] F.S. Navarra, M. Nielsen R.S. Marques de Carvalho and G. Krein, Phys. Lett. B529, 87 (2002).

[12] F.S. Navarra, M. Nielsen and M.R. Robilotta, Phys. Rev. C64, (2001) 021901 (R).

[13] Cheuk-Yin Wong, E. S. Swanson and T. Barnes, Phys. Rev. C62, 045201 (2000) and references therein. 